\title{
Approximate Solution of Fractional Nonlinear Partial Differential Equations by the Legendre Multiwavelet Galerkin Method
}

\author{
M. A. Mohamed ${ }^{1}$ and M. Sh. Torky ${ }^{2}$ \\ ${ }^{1}$ Faculty of Science, Suez Canal University Ismailia, Ismailia, Egypt \\ ${ }^{2}$ The High Institute of Administration and Computer, Port Said University, Port Said, Egypt \\ Correspondence should be addressed to M. Sh. Torky; mohamedtorky75@yahoo.com
}

Received 25 May 2013; Revised 3 January 2014; Accepted 4 January 2014; Published 3 March 2014

Academic Editor: Magdy A. Ezzat

Copyright (c) 2014 M. A. Mohamed and M. Sh. Torky. This is an open access article distributed under the Creative Commons Attribution License, which permits unrestricted use, distribution, and reproduction in any medium, provided the original work is properly cited.

\begin{abstract}
The Legendre multiwavelet Galerkin method is adopted to give the approximate solution for the nonlinear fractional partial differential equations (NFPDEs). The Legendre multiwavelet properties are presented. The main characteristic of this approach is using these properties together with the Galerkin method to reduce the NFPDEs to the solution of nonlinear system of algebraic equations. We presented the numerical results and a comparison with the exact solution in the cases when we have an exact solution to demonstrate the applicability and efficiency of the method. The fractional derivative is described in the Caputo sense.
\end{abstract}

\section{Introduction}

Nowadays, fractional differential equations have garnered a great deal of attention and appreciation recently due to its ability to provide an accurate description of different nonlinear phenomena. The process of development of models based on fractional order differential systems has lately gained popularity in the investigation of dynamical systems. The advantage of fractional order systems is that they allow greater degrees of freedom in the model. The field of chaos has also snatched the attention of the researchers and this contributes to a large amount of the current research these days.

In recent decades, fractional calculus has found diverse applications in different scientific and technological fields [15], such as thermal engineering, acoustics, electromagnetism, control, robotics, viscoelasticity, diffusion, edge detection, turbulence, signal processing, information sciences, communications, and many other physical processes and also in medical sciences. Fractional differential equations (FDEs) have also been applied in modeling many physical and engineering problems and fractional differential equations in nonlinear dynamic $[6,7]$. The importance of getting approximate and exact solutions of nonlinear fractional differential equations in mathematics and physics remains an important problem that requires to discover new methods of approximate and exact solutions. However, finding exact solutions to these nonlinear fractional differential equations are difficult to obtain it [8]. Therefore, the numerical methods used to deal with these equations [9] and they have largely been using some semianalytical techniques to solve these equations such as, differential transform method [10-17], Adomian decomposition method [18-21], Laplace decomposition method [22-24], homotopy perturbation method [25-29], and variational iteration method [30-32]. The majority of these methods have shortcomings inbuilt such as calculating Adomian's polynomials, the Lagrange multiplier, mixed results, and the large computational work.

The aim of this paper is to expand the application of Legendre multiwavelet Galerkin method to provide approximate solutions for initial value problems of fractional nonlinear partial differential equations and to make comparison with that obtained by other numerical methods. 


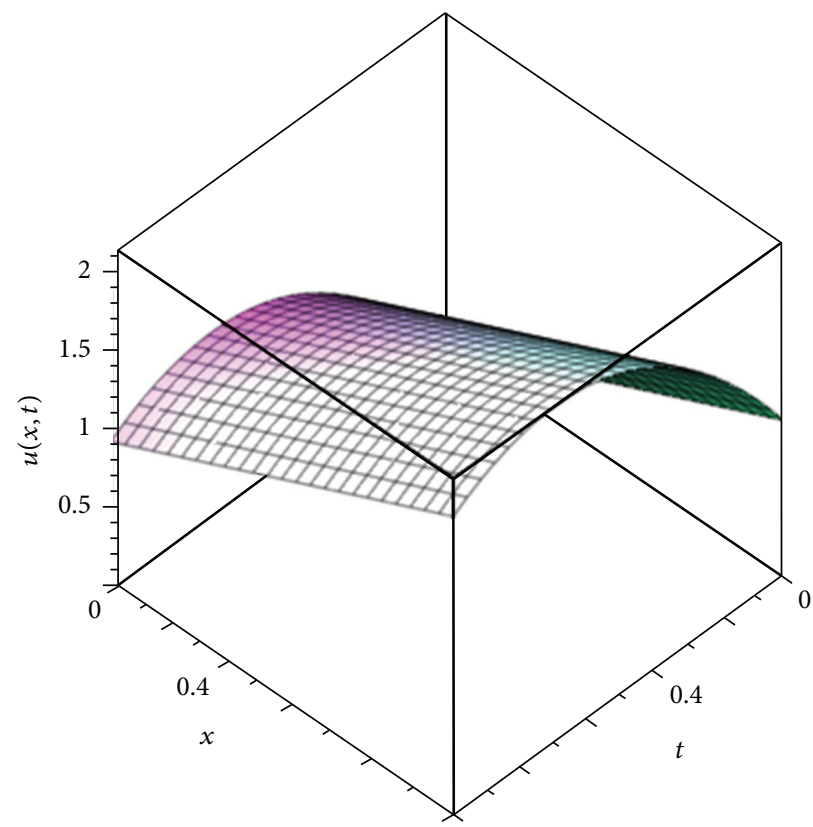

(a)

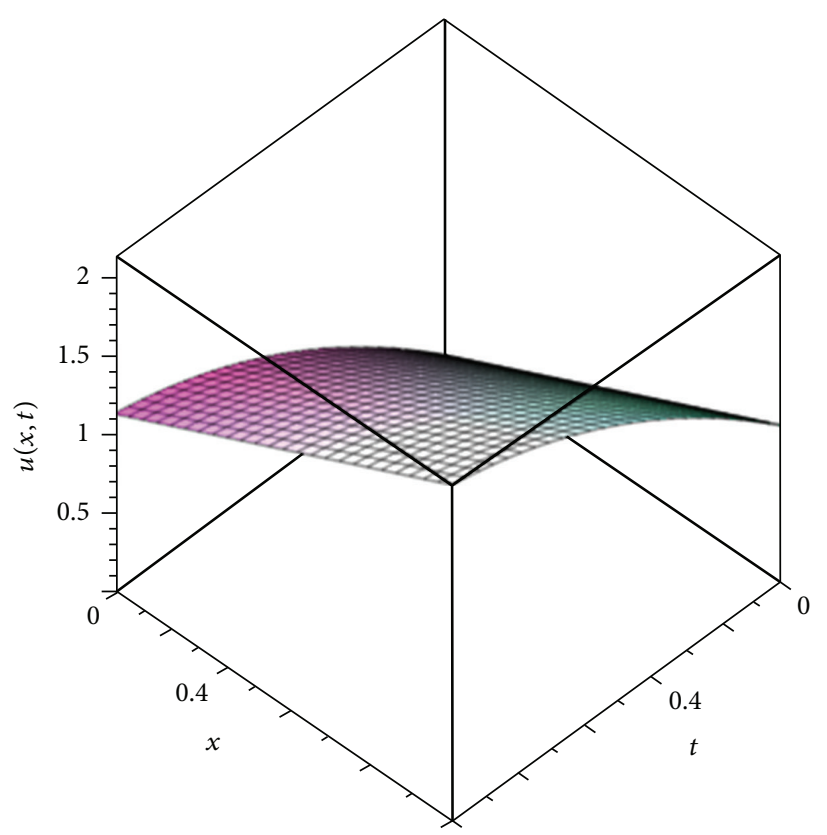

(c)

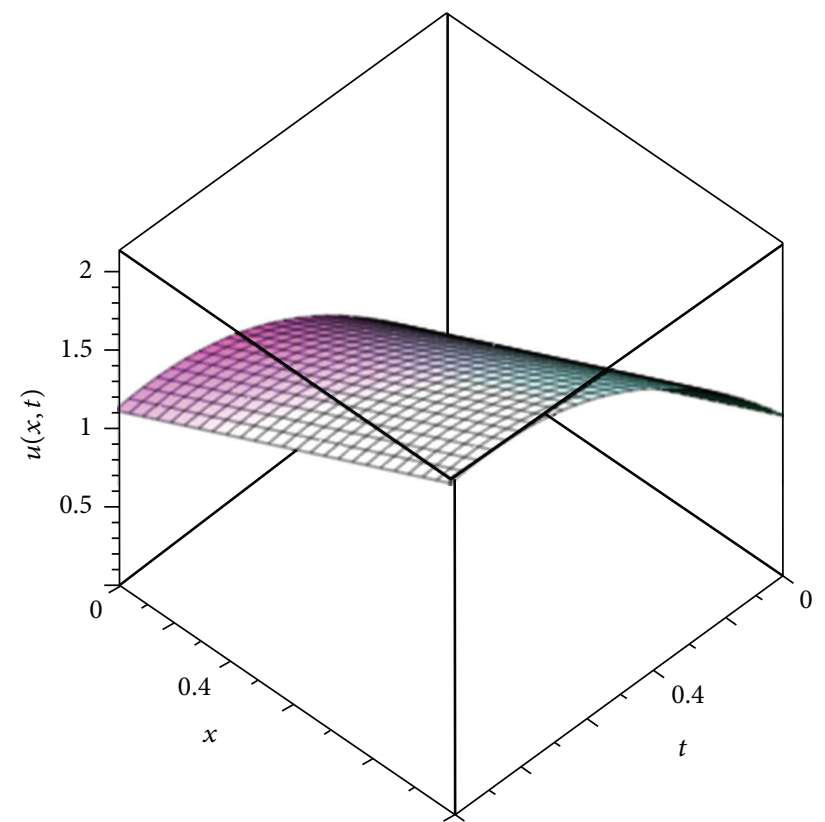

(b)

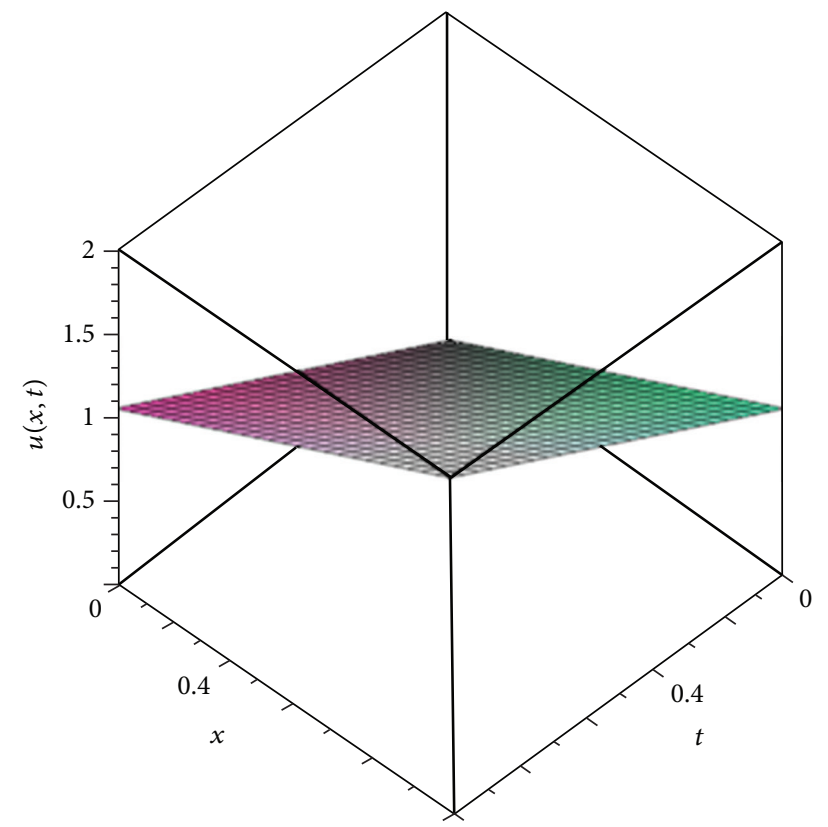

(d)

Figure 1: (a) Plot of $u(x, t)$ with respect to $x$ and $t$ at $\alpha=1 / 3$. (b) Plot of $u(x, t)$ with respect to $x$ and $t$ at $\alpha=1 / 2$. (c) Plot of $u(x, t)$ with respect to $x$ and $t$ at $\alpha=2 / 3$. (d) Plot of $u(x, t)$ with respect to $x$ and $t$ at $\alpha=1$.

\section{Preliminaries and Notations}

In this section, we give the definition of the RiemannLiouville fractional derivative and fractional integral with some basic properties.

Definition 1. The left sided Riemann-Liouville fractional integral of order $\mu \geq 0$, [33-35] of a function $f \in C_{\alpha}, \alpha \geq-1$, is defined as

$$
I^{\mu} f(t)= \begin{cases}\frac{1}{\Gamma(\mu)} \int_{0}^{t} \frac{f(\tau)}{(t-\tau)^{1-\mu}} d \tau, & \mu>0, t>0, \\ f(t) & \mu=0 .\end{cases}
$$

Definition 2. The (left sided) Caputo fractional derivative of $f, f \in C_{-1}^{m}, m \in I N \cup\{0\}$, is defined as [33]

$$
D_{*}^{\mu} f(t)=\frac{\partial^{\mu} f(t)}{\partial t^{\mu}}= \begin{cases}I^{m-\mu}\left[\frac{\partial^{m} f(t)}{\partial t^{m}}\right], & \mu>0, t>0, \\ \frac{\partial^{m} f(t)}{\partial t^{m}} & \mu=m .\end{cases}
$$




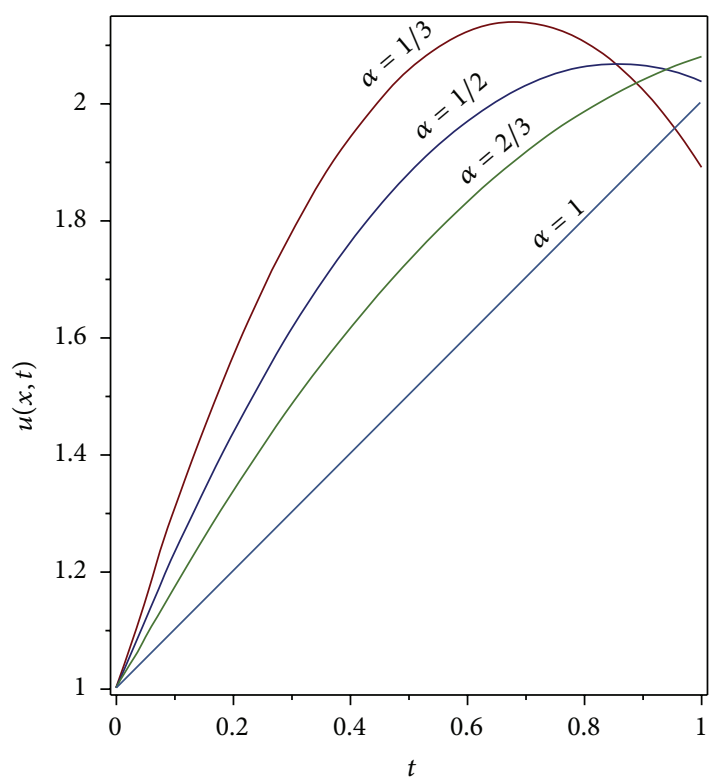

FIGURE 2: Plot of $u(x, t)$ for various values of $t$ and different values of $\alpha, x=1$.

Note that $[33-35]$

(i)

$$
I_{t}^{\mu} f(x, t)=\frac{1}{\Gamma(\mu)} \int_{0}^{t} \frac{f(x, s)}{(t-s)^{1-\pi}} d \tau, \quad \mu>0, t>0,
$$

(ii)

$$
D_{*}^{\mu} f(x, t)=I_{t}^{m-\mu}\left[\frac{\partial^{m} f(x, t)}{\partial t^{m}}\right], \quad m-1<\mu \leq m,
$$

(iii)

$$
I^{\mu} t^{\gamma}=\frac{\Gamma(\gamma+1)}{\Gamma(\gamma+\mu+1)} t^{\gamma+\mu}, \quad \mu>0, \quad \gamma>-1, t>0 .
$$

\section{Properties of Legendre Multiwavelets}

3.1. Wavelets $[35,36]$. Wavelets constitute a family of functions constructed from dilation and translation of a single function called the mother wavelet. When the dilation parameter $a$ and the translation parameter $b$ vary continuously we have the following family of continuous wavelets:

$$
\psi_{a, b}(t)=|a|^{-1 / 2} \psi\left(\frac{t-b}{a}\right), \quad a, b \in \mathbb{R}, a \neq 0 .
$$

If we restrict the parameters $a$ and $b$ to discrete values as $a=$ $a_{0}{ }^{-k}, b=n b_{0} a_{0}{ }^{-k}, a_{0}>1, b_{0}>0$, and $n, k \in \mathbb{N}$ we have the following family of discrete wavelets:

$$
\psi_{k, n}(t)=|a|^{k / 2} \psi\left(a_{0}{ }^{k} t-n b_{0}\right),
$$

where $\psi_{k, n}(t)$ form a wavelet basis for $L^{2}(R)$. In particular, when $a_{0}=2$ and $b_{0}=1$ then $\psi_{k, n}(t)$ forms an orthonormal basis [35].
3.2. Legendre Multiwavelets [36]. Legendre multiwavelets $\psi_{n m}(t)=\psi(k, n, m, t)$ have four arguments; $n, n=0,1$, $2, \ldots, 2^{k}-1, k$ can assume any positive integer; $m$ is the order for Legendre polynomials and $t$ is the normalized time. They are defined on the interval $[0,1]$

$$
\begin{aligned}
& \psi_{n m}(t) \\
& \qquad= \begin{cases}\sqrt{2 m+1} 2^{k / 2} P_{m}\left(2^{k} t-n\right), & \text { for } \frac{n}{2^{k}} \leq t \leq \frac{n+1}{2^{k}} \\
0, & \text { otherwise, }\end{cases}
\end{aligned}
$$

where $m=0,1, \ldots, M-1, n=0,1,2, \ldots, 2^{k}-1$. The coefficient $\sqrt{2 m+1}$ is for orthonormality; $P_{m}(t)$ are the wellknown shifted Legendre polynomials of order $m$ which are defined on the interval $[0,1]$ and can be determined with the aid of the following recurrence formula:

$$
\begin{aligned}
& P_{0}(t)=1, \quad P_{1}(t)=2 t-1, \\
P_{m+1}(t)= & \left(\frac{2 m+1}{m+1}\right)(2 t-1) P_{m}(t) \\
& -\left(\frac{m}{m+1}\right) P_{m-1}(t), \quad m=1,2,3, \ldots
\end{aligned}
$$

Also the two-dimensional Legendre multiwavelet is defined as

$$
\begin{aligned}
& \psi_{n_{1} m_{1} n_{2} m_{2}}(x, t) \\
& =\left\{\begin{array}{r}
A P_{m_{1}}\left(2^{k_{1}} x-n_{1}\right) P_{m_{2}}\left(2^{k_{2}} t-n_{2}\right), \\
\text { for } \frac{n_{1}}{2^{k_{1}}} \leq x \leq \frac{n_{1}+1}{2^{k_{1}}} \\
\frac{n_{2}}{2^{k_{2}}} \leq t \leq \frac{n_{2}+1}{2^{k_{2}}} \\
0, \quad \text { otherwise }
\end{array}\right.
\end{aligned}
$$

where $A=\sqrt{\left(2 m_{1}+1\right)\left(2 m_{2}+1\right)} 2^{\left(k_{1}+k_{2}\right) / 2}, n_{1}$ and $n_{2}$ are defined similarly to $n, k_{1}$ and $k_{2}$ can assume any positive integer, $m_{1}$ and $m_{2}$ are the order for the Legendre polynomials, and $\psi_{n_{1} m_{1} n_{2} m_{2}}(x, t)$ forms a basis for $L^{2}([0,1] \times[0,1])$.

3.3. Function Approximation. A function $f(x, t)$ defined over $[0,1] \times[0,1]$ can expand as

$$
f(x, t)=\sum_{n=1}^{\infty} \sum_{i=0}^{\infty} \sum_{l=1}^{\infty} \sum_{j=0}^{\infty} c_{n, i, l, j} \psi_{n, i}(x) \psi_{l, j}(t)
$$

If the infinite series in (11) is truncated, it can be written as

$$
\begin{aligned}
f(x, t) & =\sum_{n=1}^{2^{k_{1}-1}} \sum_{i=0}^{N} \sum_{l=1}^{2^{k_{2}-1}} \sum_{j=0}^{M} c_{n, i, l, j} \psi_{n, i}(x) \psi_{l, j}(t) \\
& =\Psi^{T}(x) F \Psi(t),
\end{aligned}
$$




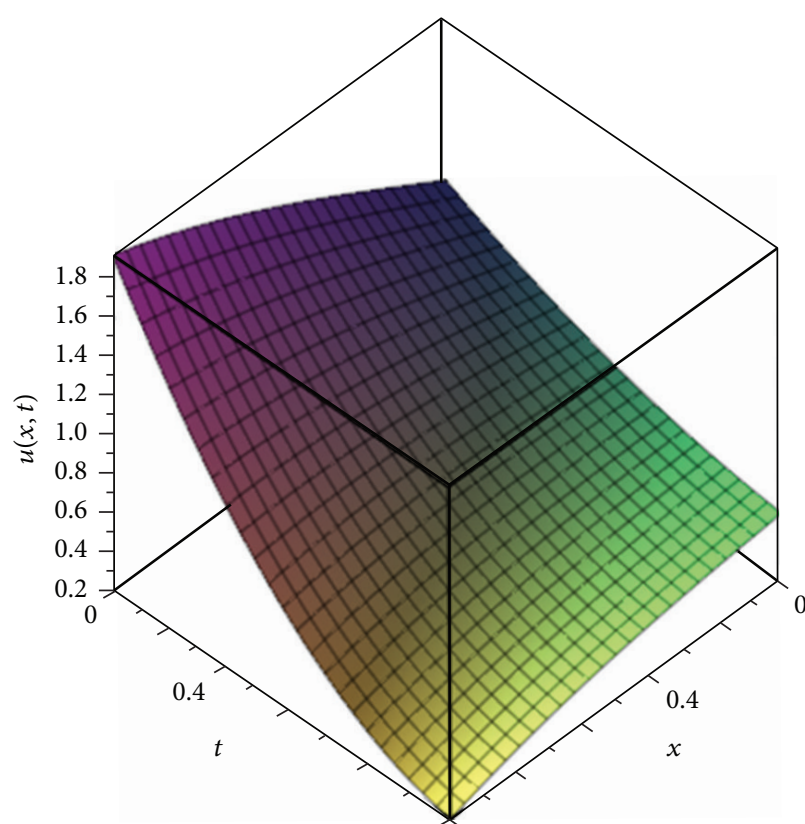

(a)

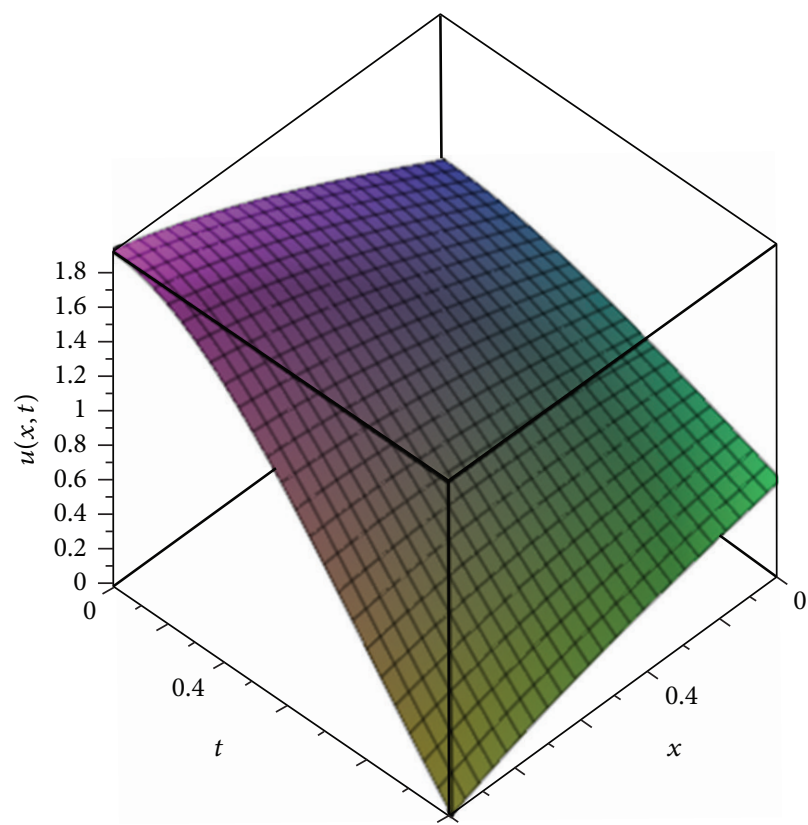

(c)

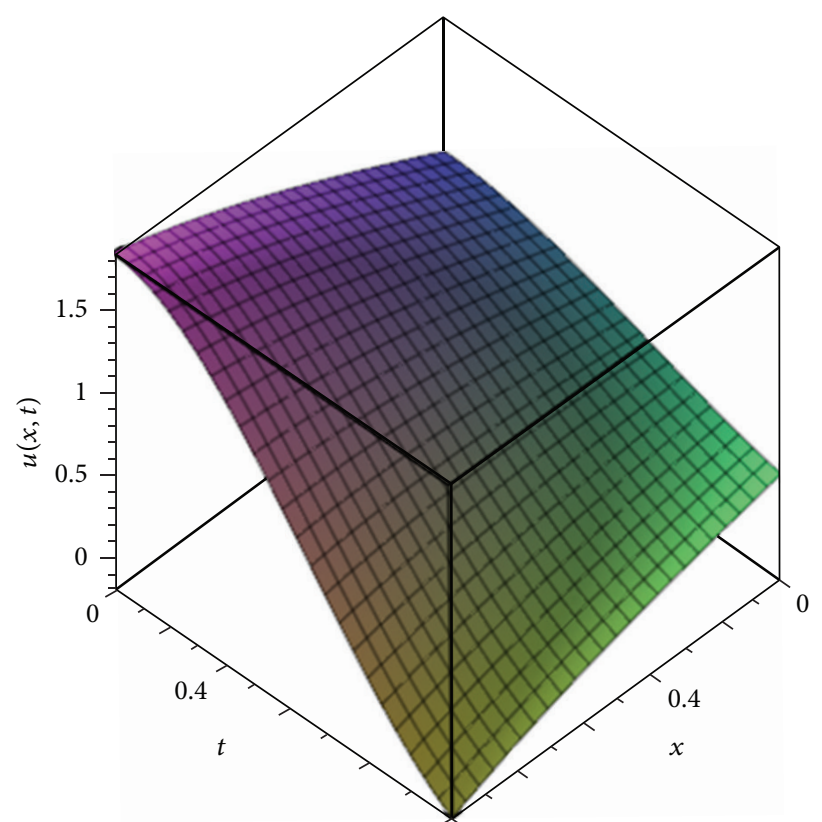

(b)

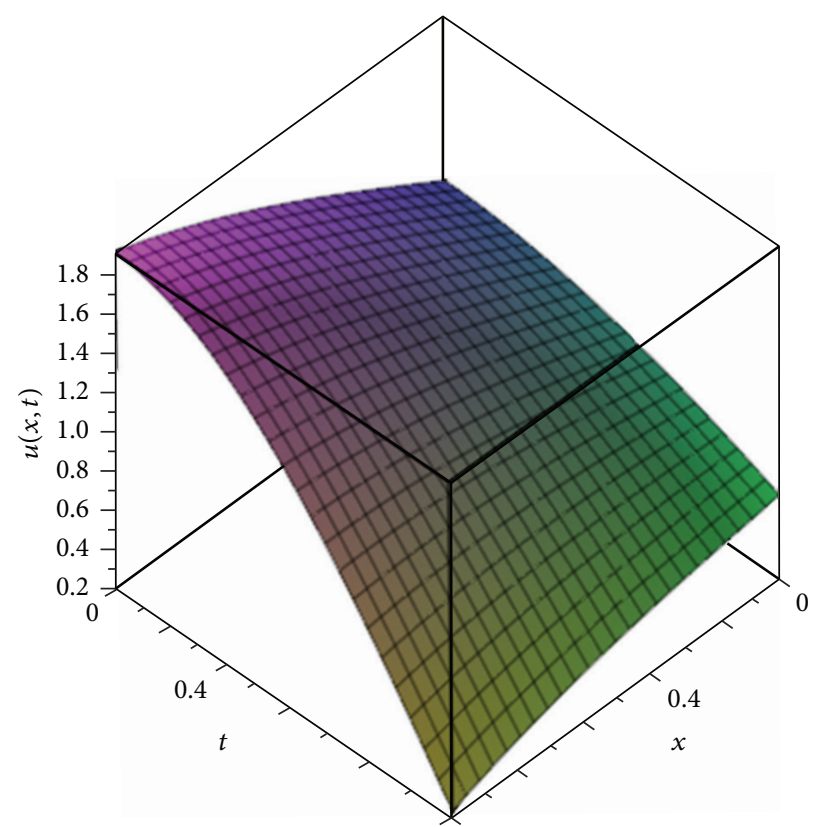

(d)

Figure 3: (a) Plot of $u(x, t)$ with respect to $x$ and $t$ at $\alpha=1$. (b) Plot of $u(x, t)$ with respect to $x$ and $t$ at $\alpha=1.5$. (c) Plot of $u(x, t)$ with respect to $x$ and $t$ at $\alpha=1.75$. (d) Plot of $u(x, t)$ with respect to $x$ and $t$ at $\alpha=2$.

where $\Psi(x)$ and $\Psi(t)$ are $2^{k_{1}}\left(M_{1}+1\right) \times 1$ and $2^{k_{2}}\left(M_{2}+1\right) \times 1$ matrices, respectively, given by

$$
\begin{aligned}
\Psi(x)= & {\left[\psi_{10}(x), \ldots, \psi_{1 M_{1}}(x), \ldots, \psi_{20}(x), \ldots,\right.} \\
& \left.\psi_{2 M_{1}}(x), \ldots, \psi_{\left(2^{k_{1}}-1\right) 0}(x), \ldots, \psi_{\left(2^{k_{1}}-1\right) M_{1}}(x)\right], \\
\Psi(t)= & {\left[\psi_{10}(t), \ldots, \psi_{1 M_{1}}(t), \ldots, \psi_{20}(t), \ldots,\right.} \\
& \left.\psi_{2 M_{1}}(t), \ldots, \psi_{\left(2^{k_{1}}-1\right) 0}(t), \ldots, \psi_{\left(2^{k_{1}}-1\right) M_{1}}(t)\right] .
\end{aligned}
$$

In addition, $F$ is a $2^{k_{1}}\left(M_{1}+1\right) \times 2^{k_{2}}\left(M_{2}+1\right)$ matrix whose elements can be calculated from

$$
\iint_{0}^{1} \psi_{n i}(x) \psi_{l j}(t) f(x, t) d t d x
$$

with $n=0,1, \ldots, 2^{k_{1}}-1, i=0, \ldots, M_{1}, l=0,1, \ldots, 2^{k_{2}}-1$, $j=0, \ldots, M_{2}$. 
TABle 1: Approximate solutions for Example 3.

\begin{tabular}{|c|c|c|c|c|c|c|c|c|c|c|}
\hline \multirow{2}{*}{$t$} & \multirow{2}{*}{$x$} & \multicolumn{2}{|c|}{$\alpha=1 / 3$} & \multicolumn{2}{|c|}{$\alpha=1 / 2$} & \multicolumn{2}{|c|}{$\alpha=2 / 3$} & \multicolumn{3}{|c|}{$\alpha=1$} \\
\hline & & $u_{\mathrm{HPM}}$ & $u_{\mathrm{LEG}}$ & $u_{\mathrm{HPM}}$ & $u_{\mathrm{LEG}}$ & $u_{\mathrm{HPM}}$ & $u_{\mathrm{LEG}}$ & $u_{\mathrm{HPM}}$ & $u_{\mathrm{LEG}}$ & $u_{\mathrm{EX}}$ \\
\hline \multirow{4}{*}{0.0} & 0.25 & 0.25 & 0.25 & 0.25 & 0.25 & 0.25 & 0.25 & 0.25 & 0.25 & 0.25 \\
\hline & 0.50 & 0.50 & 0.50 & 0.50 & 0.50 & 0.50 & 0.50 & 0.50 & 0.50 & 0.50 \\
\hline & 0.75 & 0.75 & 0.75 & 0.75 & 0.75 & 0.75 & 0.75 & 0.75 & 0.75 & 0.75 \\
\hline & 1.00 & 1.00 & 1.00 & 1.00 & 1.00 & 1.00 & 1.00 & 1.00 & 1.00 & 1.00 \\
\hline \multirow{4}{*}{0.25} & 0.25 & 0.95546 & 0.93263 & 0.81419 & 0.78225 & 0.68960 & 0.66479 & 0.50 & 0.50 & 0.50 \\
\hline & 0.50 & 1.20546 & 1.18263 & 1.06419 & 1.03225 & 0.93960 & 0.91479 & 0.75 & 0.75 & 0.75 \\
\hline & 0.75 & 1.45546 & 1.43263 & 1.31419 & 1.28225 & 1.18960 & 1.16479 & 1.00 & 1.00 & 1.00 \\
\hline & 1.00 & 1.70546 & 1.68263 & 1.56419 & 1.53225 & 1.43960 & 1.41479 & 1.25 & 1.25 & 1.25 \\
\hline \multirow{4}{*}{0.50} & 0.25 & 1.13882 & 1.30877 & 1.04788 & 1.13276 & 0.94783 & 0.98312 & 0.75 & 0.75 & 0.75 \\
\hline & 0.50 & 1.38882 & 1.55877 & 1.29788 & 1.38276 & 1.19783 & 1.23312 & 1.00 & 1.00 & 1.00 \\
\hline & 0.75 & 1.63882 & 1.80877 & 1.54788 & 1.63276 & 1.44783 & 1.48312 & 1.25 & 1.25 & 1.25 \\
\hline & 1.00 & 1.88882 & 2.05877 & 1.79788 & 1.88276 & 1.69783 & 1.73312 & 1.50 & 1.50 & 1.50 \\
\hline \multirow{4}{*}{0.75} & 0.25 & 1.26745 & 1.37843 & 1.22721 & 1.30152 & 1.16441 & 1.20499 & 1.00 & 1.00 & 1.00 \\
\hline & 0.50 & 1.51745 & 1.62843 & 1.47721 & 1.55152 & 1.41441 & 1.45499 & 1.25 & 1.25 & 1.25 \\
\hline & 0.75 & 1.76745 & 1.87843 & 1.72721 & 1.80152 & 1.66441 & 1.70499 & 1.50 & 1.50 & 1.50 \\
\hline & 1.00 & 2.01745 & 2.12843 & 1.97721 & 2.05152 & 1.91441 & 1.95499 & 1.75 & 1.75 & 1.75 \\
\hline \multirow{4}{*}{1.00} & 0.25 & 1.36985 & 1.14160 & 1.37838 & 1.28854 & 1.35773 & 1.33039 & 1.25 & 1.25 & 1.25 \\
\hline & 0.50 & 1.61985 & 1.39160 & 1.62838 & 1.53854 & 1.60773 & 1.58039 & 1.50 & 1.50 & 1.50 \\
\hline & 0.75 & 1.86985 & 1.64160 & 1.87838 & 1.78854 & 1.85773 & 1.83039 & 1.75 & 1.75 & 1.75 \\
\hline & 1.00 & 2.11985 & 1.89160 & 2.12838 & 2.03854 & 2.10773 & 2.08039 & 2.00 & 2.00 & 2.00 \\
\hline
\end{tabular}

TABLE 2: Approximate solutions for Example 4.

\begin{tabular}{|c|c|c|c|c|c|}
\hline \multirow{2}{*}{$t$} & \multirow{2}{*}{$x$} & $\alpha=1$ & $\alpha=1.5$ & 1.75 & $\alpha=2$ \\
\hline & & $u_{\mathrm{LEG}}$ & $u_{\mathrm{LEG}}$ & $u_{\mathrm{LEG}}$ & $u_{\mathrm{LEG}}$ \\
\hline \multirow{4}{*}{0.25} & 0.25 & 0.918665978 & 1.132388298 & 1.164570723 & 1.188858256 \\
\hline & 0.50 & 1.023903100 & 1.316468982 & 1.361000156 & 1.394880289 \\
\hline & 0.75 & 1.099331862 & 1.470741307 & 1.527621230 & 1.571093962 \\
\hline & 1.00 & 1.132379628 & 1.582632636 & 1.651861308 & 1.704926639 \\
\hline \multirow{4}{*}{0.50} & 0.25 & 0.670529477 & 0.862842686 & 0.954935119 & 1.028617931 \\
\hline & 0.50 & 0.683586761 & 0.936088923 & 1.063084555 & 1.165329668 \\
\hline & 0.75 & 0.666835687 & 0.979526801 & 1.141425631 & 1.272233047 \\
\hline & 1.00 & 0.607703616 & 0.980583683 & 1.177385711 & 1.336755430 \\
\hline \multirow{4}{*}{0.75} & 0.25 & 0.502994454 & 0.552019181 & 0.676793302 & 0.7897781568 \\
\hline & 0.50 & 0.458476525 & 0.501019780 & 0.671719555 & 0.8269013752 \\
\hline & 0.75 & 0.384150235 & 0.420212023 & 0.636837450 & 0.8342162344 \\
\hline & 1.00 & 0.267442949 & 0.297023267 & 0.559574347 & 0.799150097 \\
\hline \multirow{4}{*}{1.00} & 0.25 & 0.416060911 & 0.313169842 & 0.388441429 & 0.4954341090 \\
\hline & 0.50 & 0.348572389 & 0.173995974 & 0.272945982 & 0.415723105 \\
\hline & 0.75 & 0.251275507 & 0.005013748 & 0.127642174 & 0.306203742 \\
\hline & 1.00 & 0.111597629 & -0.206349475 & -0.060042629 & 0.154303383 \\
\hline
\end{tabular}

\section{Solution of Nonlinear Fractional Partial Differential Equations}

Consider the nonlinear fractional partial differential equation

$$
D_{t}^{\alpha} u=N(u)+g(x, t), \quad m<\alpha<m+1, m \geq 0,
$$

with initial condition $u(x, 0)=f(x)$.
Let

$$
F(u)=D_{t}^{\alpha} u-N(u)-g(x, t) .
$$

A Galerkin approximation to (16) is constructed as follows. The approximation $u_{N M}$ is sought in the form of the truncated series 
TABLE 3: Numerical values when $\alpha=0.5$.

\begin{tabular}{|c|c|c|c|c|c|c|}
\hline$t$ & $x$ & $u_{\mathrm{GDTM}}$ & $u_{\mathrm{HPM}}$ & $u_{\mathrm{VIM}}$ & $u_{\mathrm{ADM}}$ & $u_{\text {Leg }}$ \\
\hline \multirow{4}{*}{0.2} & 0.25 & 0.123635 & 0.104573 & 0.103750 & 0.112844 & 0.092812 \\
\hline & 0.50 & 0.247270 & 0.209146 & 0.207499 & 0.225688 & 0.185624 \\
\hline & 0.75 & 0.370905 & 0.313720 & 0.311249 & 0.311249 & 0.278436 \\
\hline & 1.00 & 0.494540 & 0.418293 & 0.414999 & 0.451375 & 0.371248 \\
\hline \multirow{4}{*}{0.4} & 0.25 & 0.177148 & 0.177229 & 0.172012 & 0.164004 & 0.161241 \\
\hline & 0.50 & 0.354295 & 0.354458 & 0.344025 & 0.328008 & 0.322483 \\
\hline & 0.75 & 0.531443 & 0.531686 & 0.516037 & 0.492011 & 0.483725 \\
\hline & 1.00 & 0.708590 & 0.708915 & 0.688050 & 0.656015 & 0.644967 \\
\hline \multirow{4}{*}{0.6} & 0.25 & 0.226965 & 0.230500 & 0.215641 & 0.243862 & 0.205288 \\
\hline & 0.50 & 0.453931 & 0.461000 & 0.431283 & 0.487721 & 0.410577 \\
\hline & 0.75 & 0.680896 & 0.691499 & 0.646924 & 0.731581 & 0.615866 \\
\hline & 1.00 & 0.907861 & 0.921999 & 0.862566 & 0.975441 & 0.821155 \\
\hline
\end{tabular}

TABLE 4: Numerical values when $\alpha=0.75$.

\begin{tabular}{|c|c|c|c|c|c|c|}
\hline$t$ & $x$ & $u_{\mathrm{GDTM}}$ & $u_{\mathrm{HPM}}$ & $u_{\mathrm{VIM}}$ & $u_{\mathrm{ADM}}$ & $u_{\text {Leg }}$ \\
\hline \multirow{4}{*}{0.2} & 0.25 & 0.080835 & 0.078306 & 0.077933 & 0.078787 & 0.070083 \\
\hline & 0.50 & 0.161671 & 0.156612 & 0.155865 & 0.157574 & 0.140166 \\
\hline & 0.75 & 0.242596 & 0.234919 & 0.233798 & 0.236361 & 0.210249 \\
\hline & 1.00 & 0.323342 & 0.313225 & 0.311730 & 0.315148 & 0.280332 \\
\hline \multirow{4}{*}{0.4} & 0.25 & 0.135446 & 0.136806 & 0.134855 & 0.128941 & 0.129648 \\
\hline & 0.50 & 0.270892 & 0.273612 & 0.269710 & 0.257881 & 0.259297 \\
\hline & 0.75 & 0.406338 & 0.410418 & 0.404565 & 0.386821 & 0.388945 \\
\hline & 1.00 & 0.541784 & 0.547225 & 0.539420 & 0.515762 & 0.518594 \\
\hline \multirow{4}{*}{0.6} & 0.25 & 0.185529 & 0.185146 & 0.179990 & 0.177238 & 0.178696 \\
\hline & 0.50 & 0.371057 & 0.370292 & 0.359979 & 0.354477 & 0.357393 \\
\hline & 0.75 & 0.556586 & 0.555437 & 0.539969 & 0.531715 & 0.536089 \\
\hline & 1.00 & 0.742114 & 0.740583 & 0.719958 & 0.7089541 & 0.714786 \\
\hline
\end{tabular}

$$
\begin{aligned}
& u_{N M}(x, t) \\
&=\left\{\begin{array}{rr}
\sum_{n=1}^{2^{k_{1}}} \sum_{i=0}^{N} \sum_{l=1}^{2^{k_{2}}} \sum_{j=0}^{M} t c_{n, i, l, j} \psi_{n, i}(x) \psi_{l, j}(t) & \text { for } m=0, \\
\sum_{n=1}^{2^{k_{1}}} \sum_{i=0}^{N} \sum_{l=1}^{2^{k_{2}}} \sum_{j=0}^{M} t^{2} c_{n, i, l, j} \psi_{n, i}(x) \psi_{l, j}(t) & \\
+u(x, 0)+t u_{t}(x, 0), & \text { for } m=1,
\end{array}\right.
\end{aligned}
$$

where $\psi_{i j}$ are the Legendre multiwavelet basis.

The expansion coefficients $c_{n, i, l, j}$ are determined by Galerkin equations:

$$
\left\langle F\left(u_{N M}\right), \psi_{n, i} \psi_{l, j}\right\rangle=0
$$

where $\langle\cdot\rangle$ denotes inner product defined as

$$
\begin{aligned}
&\langle F\left.\left(u_{N M}\right), \psi_{n, i} \psi_{l, j}\right\rangle \\
& \quad=\iint_{0}^{1} F\left(u_{N M}\right)(x, t) \psi_{n i}(x) \psi_{l j}(t) d t d x .
\end{aligned}
$$

Galerkin equations (18) give a system of $2^{k_{1}-1}(N+1) \times$ $2^{k_{2}-1}(M+1)$ equations that can be solved for the elements of

$$
\begin{aligned}
a_{n, i, l, j}, & i=0,1, \ldots, N, j=0,1, \ldots, M \\
& n=1,2, \ldots, 2^{k_{1}}, l=1,2, \ldots, 2^{k_{2}}
\end{aligned}
$$

\section{Illustrative Example}

To demonstrate the effectiveness of the method, here we consider some linear fractional partial differential equations. The Legendre wavelets are defined only for $t \in[0,1]$; we take $a=0, b=1$. The computations associated with the examples were performed using Mathematica and Maple.

Example 3. Consider the nonlinear time-fractional diffusion equation in absence of both external force and reaction term [37]

$$
\frac{\partial^{\alpha} u}{\partial t^{\alpha}}-\frac{\partial u}{\partial x}\left(u \frac{\partial u}{\partial x}\right)=0, \quad 0<\alpha \leq 1, t>0
$$

with initial condition $u(x, 0)=x$. 
TABLE 5: Numerical values when $\alpha=1$.

\begin{tabular}{|c|c|c|c|c|c|c|c|}
\hline$t$ & $x$ & $u_{\mathrm{GDTM}}$ & $u_{\mathrm{HPM}}$ & $u_{\mathrm{VIM}}$ & $u_{\mathrm{ADM}}$ & $u_{\text {Leg }}$ & $u_{\mathrm{EX}}$ \\
\hline \multirow{4}{*}{0.2} & 0.25 & 0.05 & 0.049989 & 0.050309 & 0.050000 & 0.05 & 0.05 \\
\hline & 0.50 & 0.10 & 0.099978 & 0.100619 & 0.100000 & 0.10 & 0.10 \\
\hline & 0.75 & 0.15 & 0.149968 & 0.150928 & 0.150001 & 0.15 & 0.15 \\
\hline & 1.00 & 0.20 & 0.199957 & 0.201237 & 0.200001 & 0.20 & 0.20 \\
\hline \multirow{4}{*}{0.4} & 0.25 & 0.10 & 0.099645 & 0.101894 & 0.100023 & 0.10 & 0.10 \\
\hline & 0.50 & 0.20 & 0.199290 & 0.203787 & 0.200046 & 0.20 & 0.20 \\
\hline & 0.75 & 0.30 & 0.298935 & 0.305681 & 0.300069 & 0.30 & 0.30 \\
\hline & 1.00 & 0.40 & 0.398580 & 0.407575 & 0.400092 & 0.40 & 0.40 \\
\hline \multirow{4}{*}{0.6} & 0.25 & 0.15 & 0.147158 & 0.153094 & 0.150411 & 0.15 & 0.15 \\
\hline & 0.50 & 0.30 & 0.294317 & 0.306188 & 0.300823 & 0.30 & 0.30 \\
\hline & 0.75 & 0.45 & 0.441475 & 0.459282 & 0.451234 & 0.45 & 0.45 \\
\hline & 1.00 & 0.60 & 0.588634 & 0.612376 & 0.601646 & 0.60 & 0.60 \\
\hline
\end{tabular}

TABLE 6: Numerical values when $\alpha=\{0.5,0.75,1\}$.

\begin{tabular}{|c|c|c|c|c|c|c|c|c|}
\hline \multirow{2}{*}{$t$} & \multirow{2}{*}{$x$} & \multicolumn{2}{|c|}{$\alpha=0.5$} & \multicolumn{2}{|c|}{$\alpha=0.75$} & \multicolumn{3}{|c|}{$\alpha=1$} \\
\hline & & $u_{\mathrm{HPM}}$ & $u_{\text {Leg }}$ & $u_{\mathrm{HPM}}$ & $u_{\text {Leg }}$ & $u_{\mathrm{HPM}}$ & $u_{\text {Leg }}$ & $u_{\mathrm{EX}}$ \\
\hline \multirow{4}{*}{0.2} & 0.25 & 0.243843 & 0.216416 & 0.153820 & 0.150051 & 0.09 & 0.09 & 0.09 \\
\hline & 0.50 & 0.351274 & 0.309228 & 0.232395 & 0.220134 & 0.14 & 0.14 & 0.14 \\
\hline & 0.75 & 0.458705 & 0.402040 & 0.310971 & 0.290218 & 0.19 & 0.19 & 0.19 \\
\hline & 1.00 & 0.566136 & 0.494853 & 0.389547 & 0.360301 & 0.24 & 0.24 & 0.24 \\
\hline \multirow{4}{*}{0.4} & 0.25 & 0.534552 & 0.474657 & 0.388100 & 0.363967 & 0.26 & 0.26 & 0.26 \\
\hline & 0.50 & 0.667613 & 0.635899 & 0.514376 & 0.493616 & 0.36 & 0.36 & 0.36 \\
\hline & 0.75 & 0.800673 & 0.797140 & 0.640652 & 0.623264 & 0.46 & 0.46 & 0.46 \\
\hline & 1.00 & 0.933734 & 0.958382 & 0.766928 & 0.752913 & 0.56 & 0.56 & 0.56 \\
\hline \multirow{4}{*}{0.6} & 0.25 & 0.934713 & 0.774722 & 0.726195 & 0.641747 & 0.51 & 0.51 & 0.51 \\
\hline & 0.50 & 1.083891 & 0.980011 & 0.890481 & 0.820443 & 0.66 & 0.66 & 0.66 \\
\hline & 0.75 & 1.233068 & 1.185300 & 1.054767 & 0.999140 & 0.81 & 0.81 & 0.81 \\
\hline & 1.00 & 1.382245 & 1.390589 & 1.219052 & 1.177837 & 0.96 & 0.96 & 0.96 \\
\hline
\end{tabular}

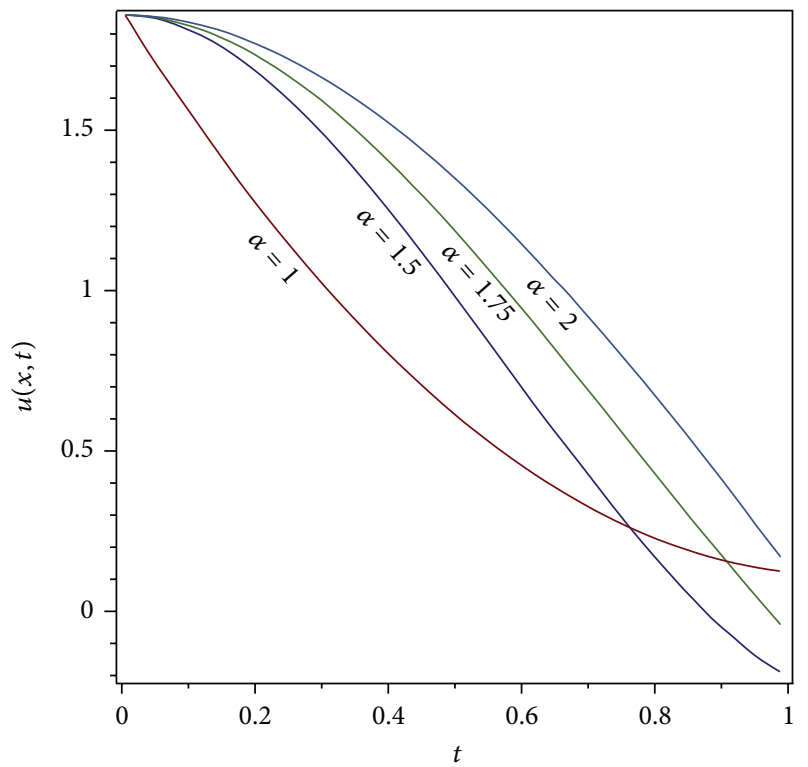

Figure 4: Plot of $u(x, t)$ for various values of $t$ and different values of $\alpha, x=1$.
We applied the method presented in this paper for $k_{1}=$ $k_{2}=0$ and $M=N=1$; from (17) we have

$$
u_{N M}(x, t)=\sum_{i=1}^{1} \sum_{j=0}^{1} t c_{0, i, 0, j} \psi_{0, i}(x) \psi_{0, j}(t)+x .
$$

Substituting (22) into (21) and using (18) and (19) we obtained the solution of (21) for different values of $\alpha=$ $\{1 / 3,1 / 2,2 / 3,1\}$. Table 1 shows the approximate solutions for (21) obtained for different values of $\alpha$ using the Legendre multiwavelet method and the Homotopy perturbation method [37]. The values of $\alpha=1$ are the only case for which we know the exact solution $u(x, t)=x+t$ and our approximate solution using Legendre multiwavelet method coincides with the approximate solution obtained using the Homotopy perturbation method [37]. It is noted that only two bases of Legendre multiwavelet and fourth-order term of Homotopy perturbation method were used in evaluating the approximate solution of Table 1. Figure 1 shows a plot of $u(x, t)$ with respect to $x$ and $t$ for different values of $\alpha=$ $\{1 / 3,1 / 2,2 / 3,1\}$. Figure 2 shows a plot of $u(x, t)$ for various values of $t$ and different values of $\alpha=\{1 / 3,1 / 2,2 / 3,1\}, x=1$. 


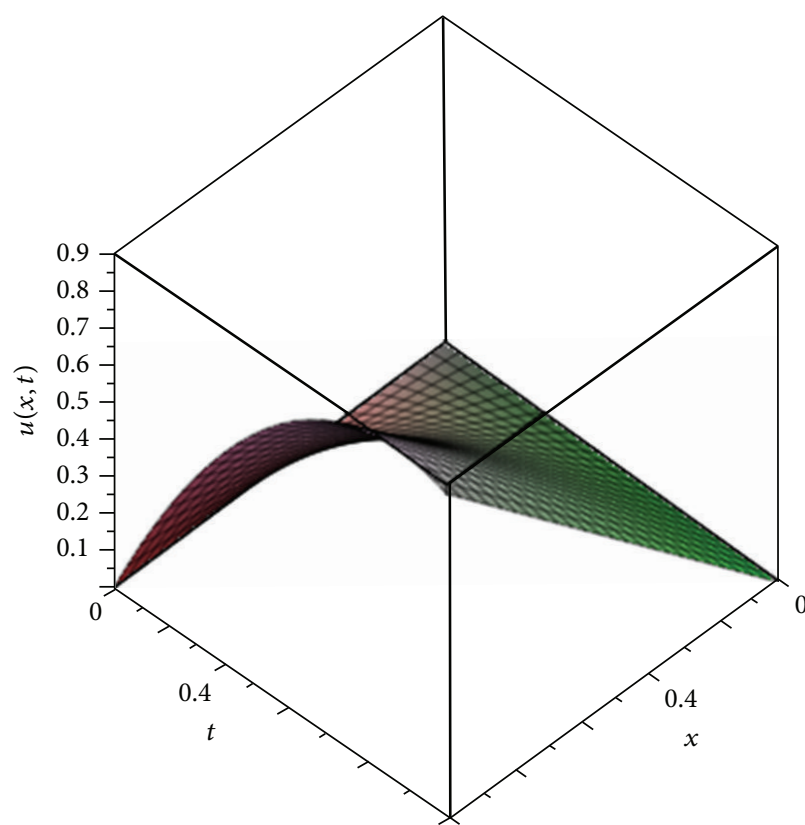

(a)

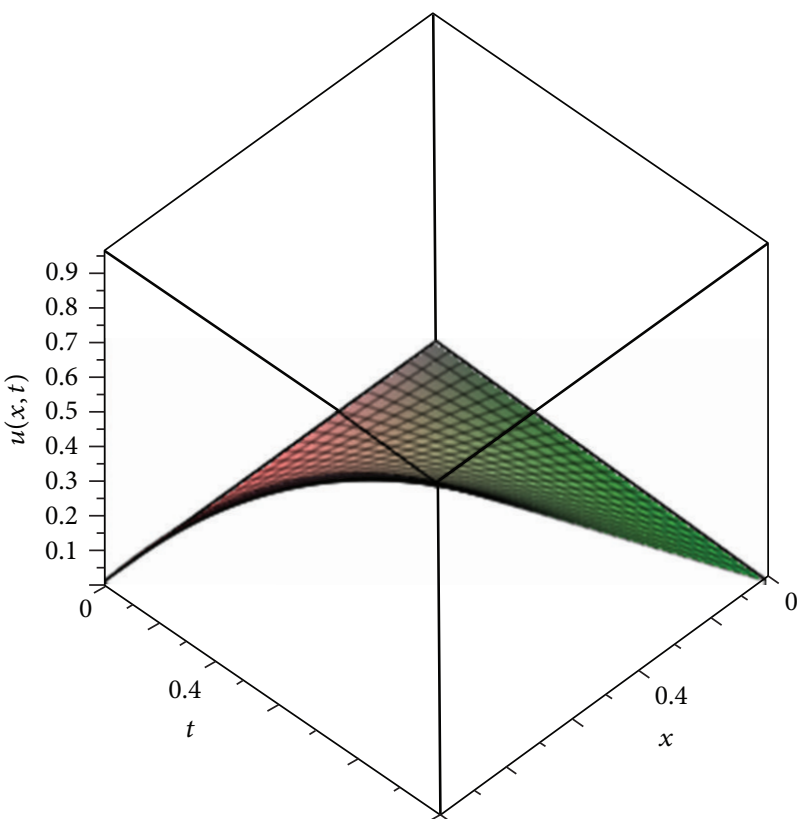

(b)

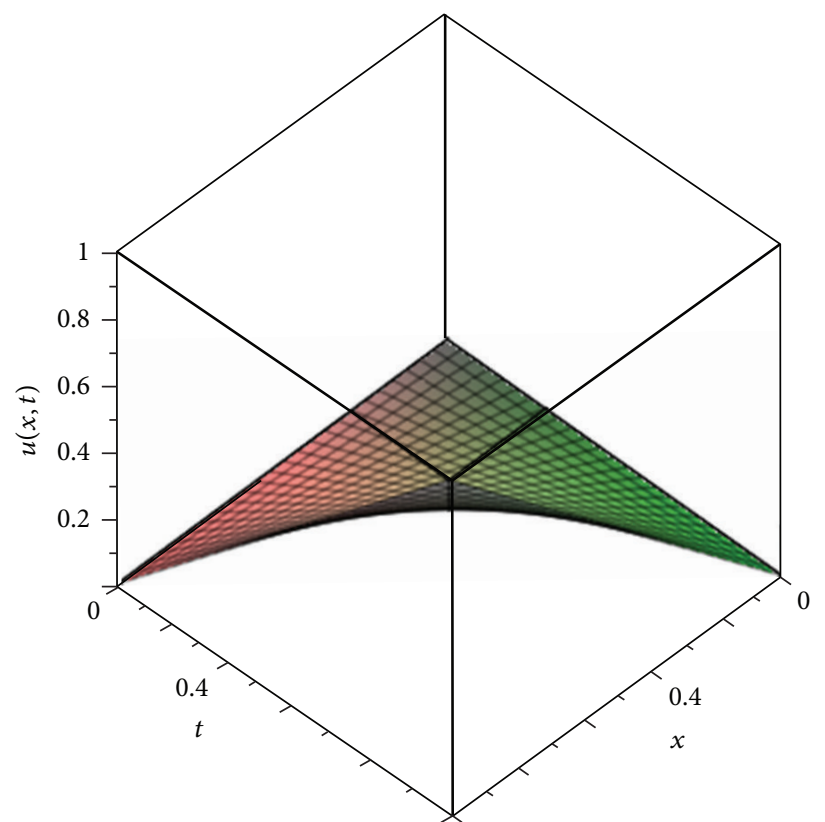

(c)

FIgURE 5: Plot of $u(x, t)$ with respect to $x$ and $t$ at (a) $\alpha=0.5$, (b) $\alpha=0.75$, and (c) $\alpha=1$.

Example 4. Consider the fractional nonlinear Klein-Gordon equation [38]

$$
\frac{\partial^{\alpha} u}{\partial t^{\alpha}}-\frac{\partial}{\partial x}\left(\frac{\partial u}{\partial x}\right)+u^{2}=0, \quad 1 \leq \alpha \leq 2, t>0
$$

with initial condition $u(x, 0)=1+\sin x, u_{t}(x, 0)=$ 0 .
We applied the method presented in this paper for $k_{1}=$ $k_{2}=0$ and $M=N=1$; from (17) we have

$$
u_{N M}(x, t)=\sum_{i=1}^{1} \sum_{j=0}^{1} t^{2} c_{0, i, 0, j} \psi_{0, i}(x) \psi_{0, j}(t)+1+\sin x .
$$

Substituting (24) into (23) and using (17) we obtained the solution of (23) for different values of $\alpha=\{1,1.5,1.75,2\}$. Table 2 shows the approximate solutions for (23) obtained for different values of $\alpha$ using the Legendre multiwavelet method. We have given the solution simulations in Figure 3 


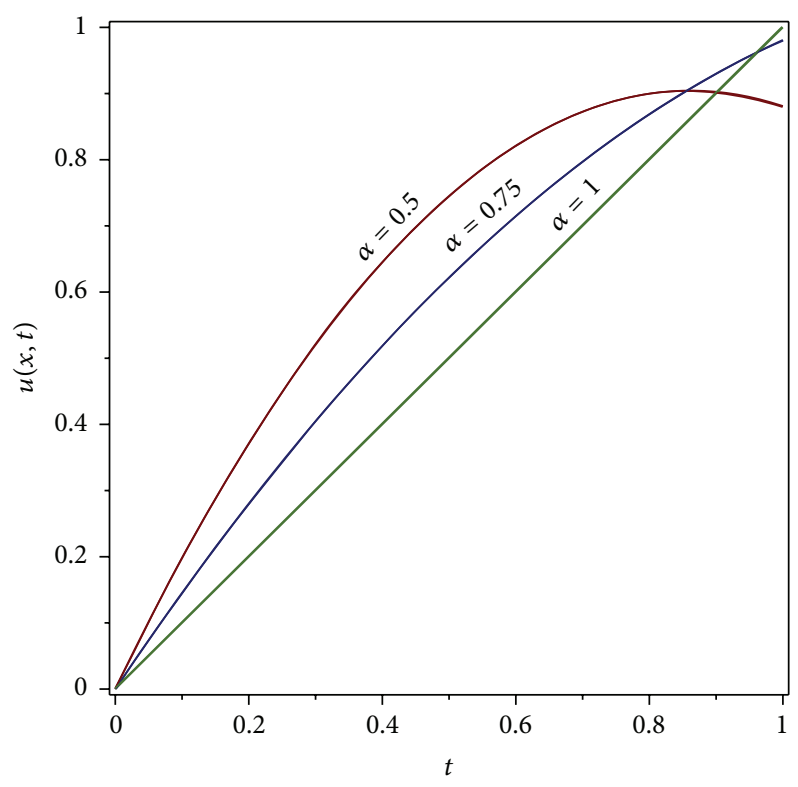

FIGURE 6: Plot of $u(x, t)$ for various values of $t$ and different values of $\alpha, x=1$.

according to different values of $\alpha$. Figure 4 shows a plot of $u(x, t)$ for various values of $t$ and different values of $\alpha, x=1$.

Example 5. Consider the following nonlinear time-fractional equation $[28,39,40]$ :

$$
\frac{\partial^{\alpha} u}{\partial t^{\alpha}}+u \frac{\partial u}{\partial x}=x+x t^{2}, \quad 0<\alpha \leq 1, t>0
$$

with initial condition $u(x, 0)=0$.

We applied the method presented in this paper for $k_{1}=$ $k_{2}=0$ and $M=N=1$; from (17) we have

$$
u_{N M}(x, t)=\sum_{i=1}^{1} \sum_{j=0}^{1} t^{2} c_{0, i, 0, j} \psi_{0, i}(x) \psi_{0, j}(t)
$$

Substituting (26) into (25) and using (17) we obtained the solution of (25) for different values of $\alpha=\{0.5,0.75,1\}$. We have given the solution simulations in Figure 5 according to different values of $\alpha$. Figure 6 shows a plot of $u(x, t)$ for various values of $t$ and different values of $\alpha, x=1$. Tables 3,4 , and 5 show the approximate solutions for (25) obtained using the Legendre multiwavelet method, the decomposition method [39], the variational iteration method [39], Homotopy perturbation method [28], and generalized differential transform method (GDTM) [40] for different values of $\alpha=$ $\{0.5,0.75,1\}$. The values of $\alpha=1$ are the only case for which we know the exact solution $u(x, t)=x t$ and Table 4 provides that our approximate solution using Legendre multiwavelet is more accurate than the approximate solution obtained using the decomposition method, Homotopy perturbation method, and the variational iteration method. In addition to that, our approximate solution using Legendre multiwavelet as the approximate solution was obtained using GDTM and exact solution.

Example 6. Consider the following time fractional advection nonhomogeneous equation $[41,42]$ :

$$
\frac{\partial^{\alpha} u}{\partial t^{\alpha}}+u \frac{\partial u}{\partial x}=2 t+x+t^{3}+x t^{2}, \quad 0<\alpha \leq 1, t>0
$$

with initial condition $u(x, 0)=0$.

We applied the method presented in this paper for $k_{1}=$ $k_{2}=0$ and $M=N=1$; from (17) we have

$$
u_{N M}(x, t)=\sum_{i=1}^{1} \sum_{j=0}^{1} t^{2} c_{0, i, 0, j} \psi_{0, i}(x) \psi_{0, j}(t)
$$

Substituting (28) into (27) and using (17) we obtained the solution of (27) for different values of $\alpha=\{0.5,0.75,1\}$. Table 6 shows the approximate solutions for (27) obtained for different values of $\alpha$ using the Legendre multiwavelet method and the Homotopy perturbation method [41]. The values of $\alpha=1$ are the only case for which we know the exact solution $u(x, t)=t^{2}+x t$ and our approximate solution using Legendre multiwavelet method coincides with the approximate solution obtained using the Homotopy perturbation method [41, 42] and the approximate solution obtained using Adomian decomposition method and variational iteration method [43]. We have given the solution simulations in Figure 7 according to different values of $\alpha=\{0.5,0.75,1\}$. Figure 8 shows a plot of $u(x, t)$ for various values of $t$ and $\alpha=\{0.5,0.75,1\}, x=1$. 


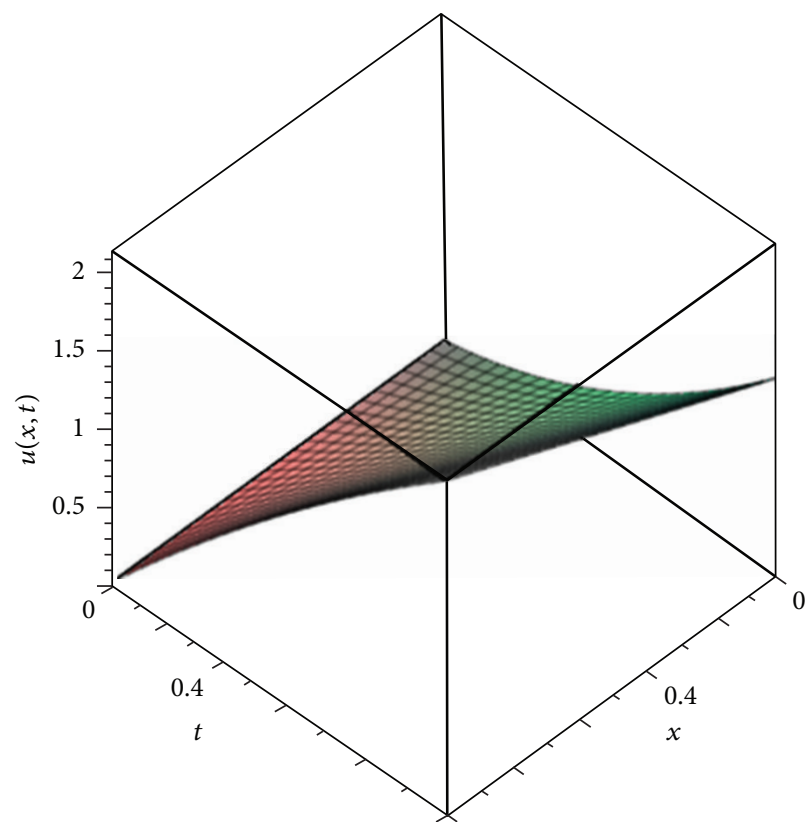

(a)

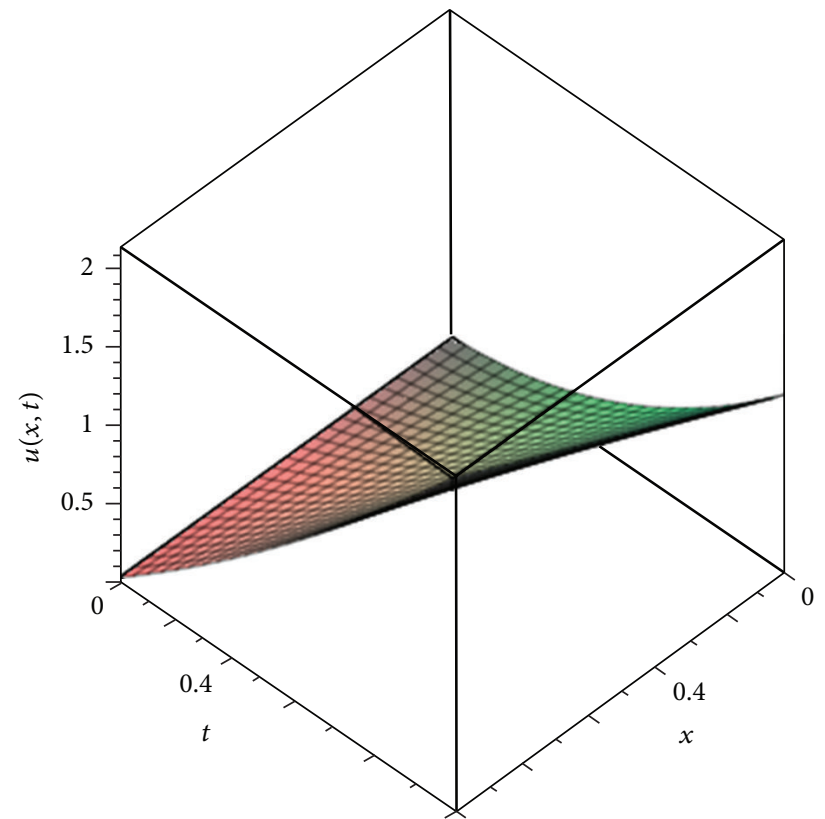

(b)

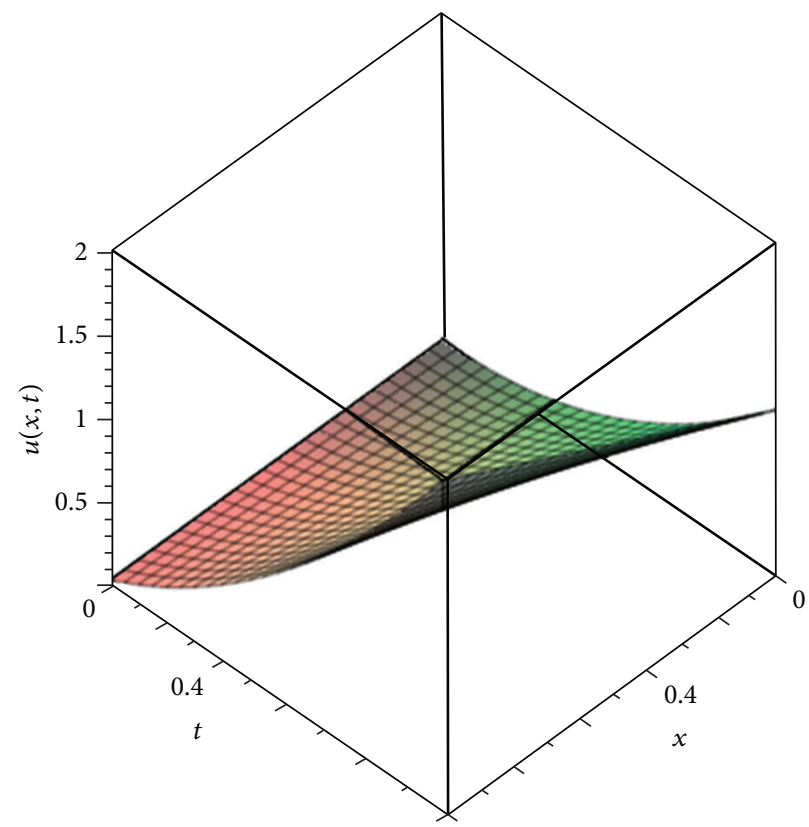

(c)

Figure 7: Plot of $u(x, t)$ with respect to $x$ and $t$ at (a) $\alpha=0.5$, (b) $\alpha=0.75$, and (c) $\alpha=1$.

\section{Conclusion}

In this study, it is shown how Legendre multiwavelet can be applied to provide approximate solutions for initial value problems of fractional nonlinear partial differential equations. The Legendre multiwavelet properties are presented. The main characteristic of this approach is using these properties together with the Galerkin method to reduce the
NFPDEs to the solution of nonlinear system of algebraic equations. In addition, we compered our results with that obtained by other numerical methods. The results show that the Legendre multiwavelet is a powerful mathematical tool for fractional nonlinear partial differential equations. We used Mathematica and Maple programs for computations in this paper. 


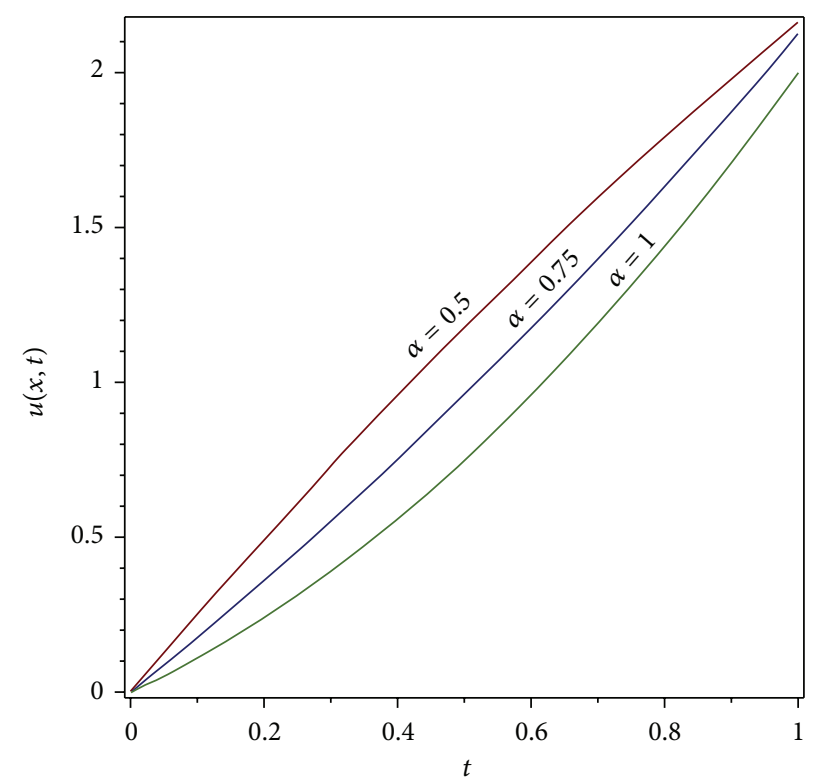

FIGURE 8: Plot of $u(x, t)$ for various values of $t$ and different values of $\alpha, x=1$.

\section{Conflict of Interests}

The authors declare that there is no conflict of interests regarding the publication of this paper.

\section{References}

[1] K. B. Oldham and J. Spanier, The Fractional Calculus, Academic Press, New York, NY, USA, 1974.

[2] F. Mainardi, "Fractional calculus: some basic problems in continuum and statistical mechanics," in Fractals and Fractional Calculus in Continuum Mechanics, vol. 378, pp. 291-348, Springer, New York, NY, USA, 1997.

[3] I. Podlubny, Fractional Differential Equations, vol. 198, Academic Press, San Diego, Calif, USA, 1999.

[4] R. Hilfer, Applications of Fractional Calculus in Physics, Academic Press, Orlando, Fla, USA, 1999.

[5] M. Dalir and M. Bashour, "Applications of fractional calculus," Applied Mathematical Sciences, vol. 4, no. 21-24, pp. 1021-1032, 2010.

[6] H. Jafari and V. Daftardar-Gejji, "Solving a system of nonlinear fractional differential equations using Adomian decomposition," Journal of Computational and Applied Mathematics, vol. 196, no. 2, pp. 644-651, 2006.

[7] J. Lu and G. Chen, "A note on the fractional-order Chen system," Chaos, Solitons and Fractals, vol. 27, no. 3, pp. 685-688, 2006.

[8] Y. Q. Liu and J. H. Ma, "Exact solutions of a generalized multifractional nonlinear diffusion equation in radical symmetry," Communications in Theoretical Physics, vol. 52, no. 5, pp. 857861, 2009.

[9] Z. A. Anastassi and T. E. Simos, "Numerical multistep methods for the efficient solution of quantum mechanics and related problems," Physics Reports, vol. 482-483, pp. 1-240, 2009.

[10] V. S. Ertürk and S. Momani, "Solving systems of fractional differential equations using differential transform method," Journal of Computational and Applied Mathematics, vol. 215, no. 1, pp. 142-151, 2008.
[11] V. S. Erturk, S. Momani, and Z. Odibat, "Application of generalized differential transform method to multi-order fractional differential equations," Communications in Nonlinear Science and Numerical Simulation, vol. 13, no. 8, pp. 1642-1654, 2008.

[12] Z. Odibat and S. Momani, "A generalized differential transform method for linear partial differential equations of fractional order," Applied Mathematics Letters, vol. 21, no. 2, pp. 194-199, 2008.

[13] Z. Odibat, S. Momani, and V. S. Erturk, "Generalized differential transform method: application to differential equations of fractional order," Applied Mathematics and Computation, vol. 197, no. 2, pp. 467-477, 2008.

[14] M. Kurulay and M. Bayram, "Approximate analytical solution for the fractional modified $\mathrm{KdV}$ by differential transform method," Communications in Nonlinear Science and Numerical Simulation, vol. 15, no. 7, pp. 1777-1782, 2010.

[15] A. K. Alomari, "A new analytic solution for fractional chaotic dynamical systems using the differential transform method," Computers \& Mathematics with Applications, vol. 61, no. 9, pp. 2528-2534, 2011.

[16] J. Liu and G. Hou, "Numerical solutions of the space- and timefractional coupled Burgers equations by generalized differential transform method," Applied Mathematics and Computation, vol. 217, no. 16, pp. 7001-7008, 2011.

[17] A. Çetinkaya and O. Kiymaz, "The solution of the timefractional diffusion equation by the generalized differential transform method," Mathematical and Computer Modelling, vol. 57, pp. 2349-2354, 2013.

[18] A. M. A. El-Sayed and M. Gaber, "The Adomian decomposition method for solving partial differential equations of fractal order in finite domains," Physics Letters A, vol. 359, no. 3, pp. 175-182, 2006.

[19] H. Jafari and V. Daftardar-Gejji, "Solving linear and nonlinear fractional diffusion and wave equations by Adomian decomposition," Applied Mathematics and Computation, vol. 180, no. 2, pp. 488-497, 2006. 
[20] Q. Wang, "Numerical solutions for fractional KdV-Burgers equation by Adomian decomposition method," Applied Mathematics and Computation, vol. 182, no. 2, pp. 1048-1055, 2006.

[21] M. Safari, D. D. Ganji, and M. Moslemi, "Application of He's variational iteration method and Adomian's decomposition method to the fractional KdV-Burgers-Kuramoto equation," Computers \& Mathematics with Applications, vol. 58, no. 11-12, pp. 2091-2097, 2009.

[22] E. Yusufoğlu, "Numerical solution of Duffing equation by the Laplace decomposition algorithm," Applied Mathematics and Computation, vol. 177, no. 2, pp. 572-580, 2006.

[23] Y. Khan, "An effective modification of the Laplace decomposition method for nonlinear equations," International Journal of Nonlinear Sciences and Numerical Simulation, vol. 10, pp. 13731376, 2009.

[24] H. Jafari, C. M. Khalique, and M. Nazari, "Application of the Laplace decomposition method for solving linear and nonlinear fractional diffusion-wave equations," Applied Mathematics Letters, vol. 24, no. 11, pp. 1799-1805, 2011.

[25] K. A. Gepreel, T. A. Nofal, and F. M. Alotaibi, "Numerical solutions for the time and space fractional nonlinear partial differential equations," Journal of Applied Mathematics, vol. 2013, Article ID 482419, 12 pages, 2013.

[26] P. K. Gupta and M. Singh, "Homotopy perturbation method for fractional Fornberg-Whitham equation," Computers \& Mathematics with Applications, vol. 61, no. 2, pp. 250-254, 2011.

[27] Q. Wang, "Homotopy perturbation method for fractional KdV equation," Applied Mathematics and Computation, vol. 190, no. 2, pp. 1795-1802, 2007.

[28] S. Momani and Z. Odibat, "Homotopy perturbation method for nonlinear partial differential equations of fractional order," Physics Letters A, vol. 365, no. 5-6, pp. 345-350, 2007.

[29] A. Yıldırım and H. Koçak, "Homotopy perturbation method for solving the space-time fractional advection-dispersion equation," Advances in Water Resources, vol. 32, pp. 1711-1716, 2009.

[30] Y. M. R, M. S. M. Noorani, and I. Hashim, "Variational iteration method for fractional heat- and wave-like equations," Nonlinear Analysis: Real World Applications, vol. 10, no. 3, pp. 1854-1869, 2009.

[31] Z. Odibat and S. Momani, “The variational iteration method: an efficient scheme for handling fractional partial differential equations in fluid mechanics," Computers \& Mathematics with Applications, vol. 58, no. 11-12, pp. 2199-2208, 2009.

[32] S. Guo, L. Mei, and Y. Li, "Fractional variational homotopy perturbation iteration method and its application to a fractional diffusion equation," Applied Mathematics and Computation, vol. 219, no. 11, pp. 5909-5917, 2013.

[33] Y. Luchko and R. Gorenflo, "An operational method for solving fractional differential equations with the Caputo derivatives," Acta Mathematica Vietnamica, vol. 24, no. 2, pp. 207-233, 1999.

[34] S. Y. Lukashchuk, "An approximate solution method for ordinary fractional differential equations with the RiemannLiouville fractional derivatives," Communications in Nonlinear Science and Numerical Simulation, vol. 19, no. 2, pp. 390-400, 2014.

[35] J. S. Gu and W. S. Jiang, "The Haar wavelets operational matrix of integration," International Journal of Systems Science, vol. 27, pp. 623-628, 1996.

[36] S. A. Yousefi, "Legendre multiwavelet Galerkin method for solving the hyperbolic telegraph equation," Numerical Methods for Partial Differential Equations, vol. 26, no. 3, pp. 535-543, 2010.
[37] S. Das, K. Vishal, and P. K. Gupta, "Solution of the nonlinear fractional diffusion equation with absorbent term and external force," Applied Mathematical Modelling, vol. 35, no. 8, pp. 39703979, 2011.

[38] M. Kurulay, "Solving the fractional nonlinear Klein-Gordon equation by means of the homotopy analysis method," Advances in Difference Equations, vol. 2012, article 187, 8 pages, 2012.

[39] Z. Odibat and S. Momani, "Numerical methods for nonlinear partial differential equations of fractional order," Applied Mathematical Modelling, vol. 32, pp. 28-39, 2008.

[40] S. Momani and Z. Odibat, "A novel method for nonlinear fractional partial differential equations: combination of DTM and generalized Taylor's formula," Journal of Computational and Applied Mathematics, vol. 220, no. 1-2, pp. 85-95, 2008.

[41] Y. Liu, "Approximate solutions of fractional nonlinear equations using homotopy perturbation transformation method," Abstract and Applied Analysis, vol. 2012, Article ID 752869, 14 pages, 2012.

[42] Y. Khan and Q. Wu, "Homotopy perturbation transform method for nonlinear equations using He's polynomials," Computers \& Mathematics with Applications, vol. 61, no. 8, pp. 19631967, 2011.

[43] A.-M. Wazwaz, "A comparison between the variational iteration method and Adomian decomposition method," Journal of Computational and Applied Mathematics, vol. 207, no. 1, pp.129136, 2007 


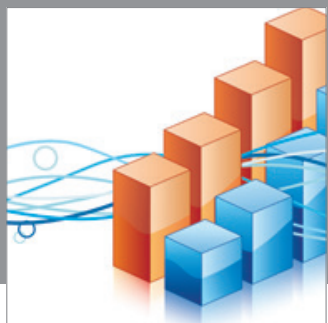

Advances in

Operations Research

mansans

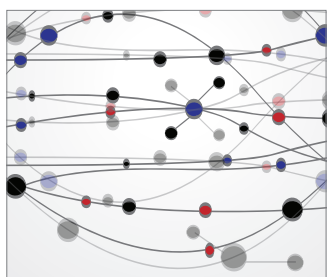

The Scientific World Journal
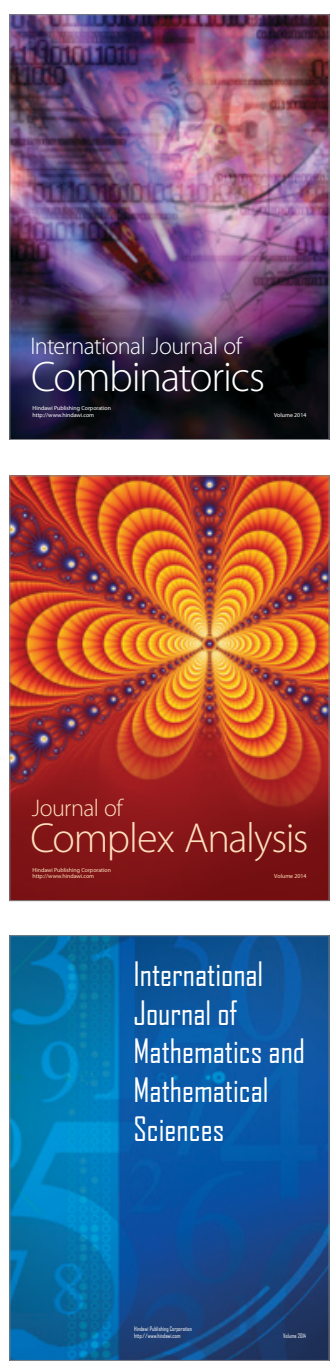
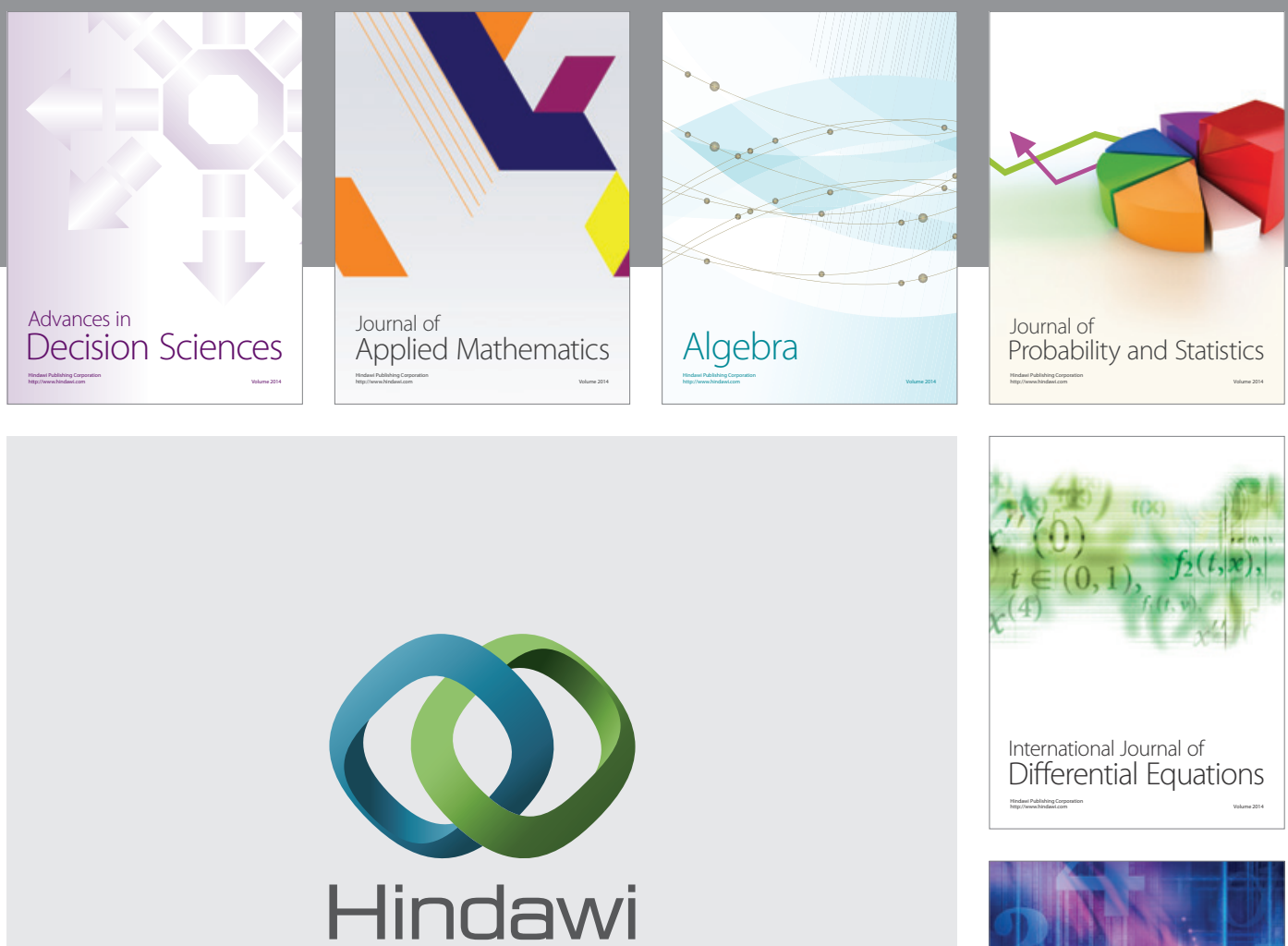

Submit your manuscripts at http://www.hindawi.com
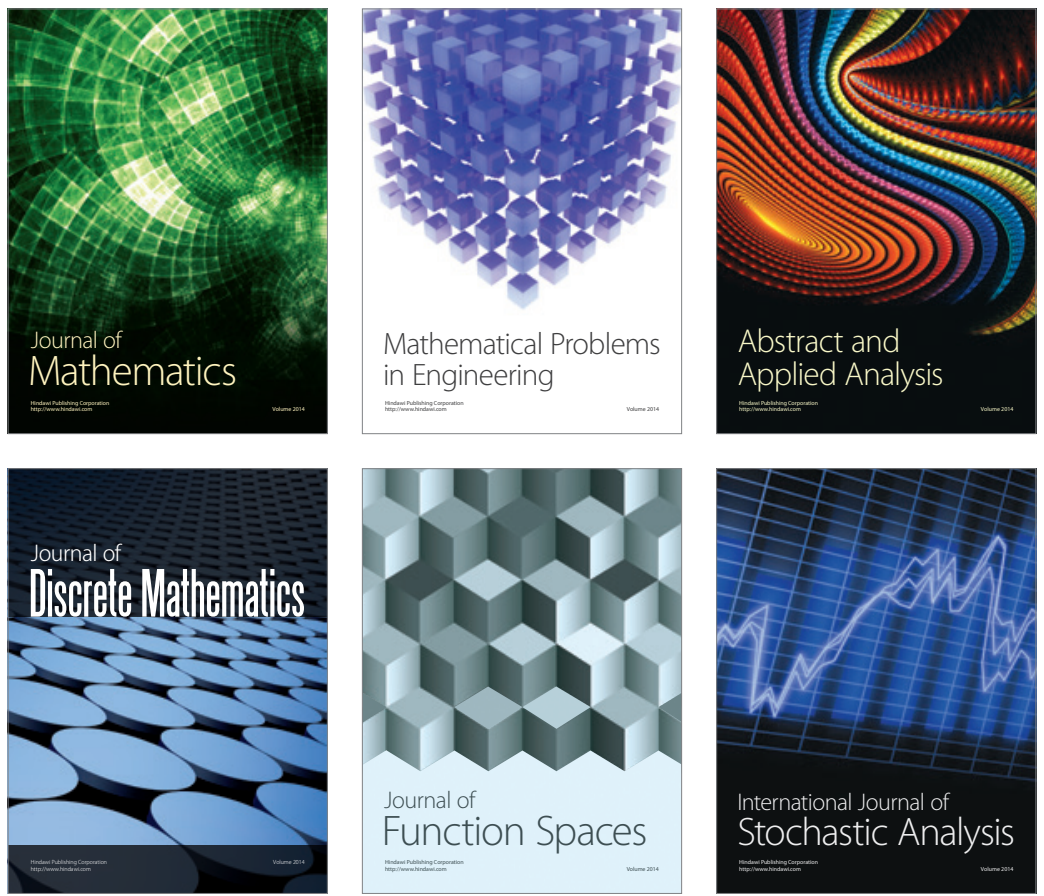

Journal of

Function Spaces

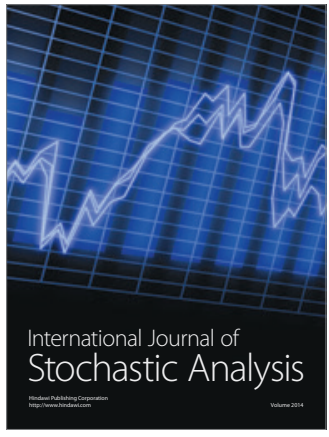

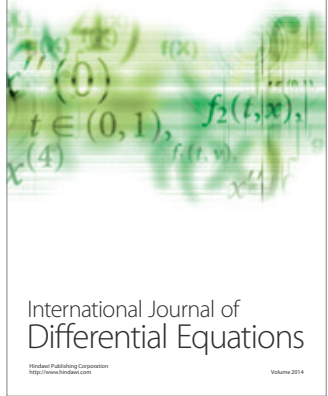
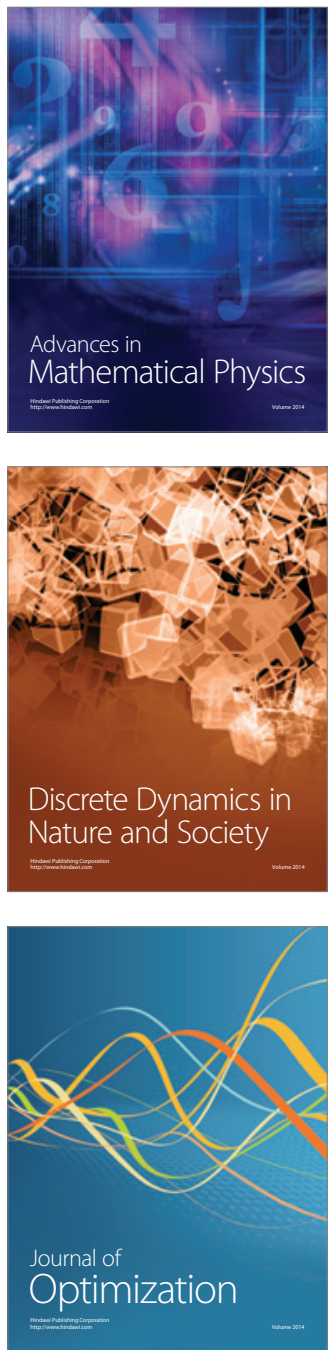